\title{
List of CGEA Underwriters
}

Lt Col. Ervan L. Amidon, USAF (Ret.)

Virginia Campbell Colgan Bruner

Drew, Grant \& Kyle Caddell

Bob \& June Campbell

Clarence A. \& Anita M. Campbell

Daniel B. Campbell

Diarmid A. Campbell

Douglass Campbell

John \& Bobbi Campbell

John A. L. Campbell

Lee E. Campbell

Leonard B. Campbell

Niall Campbell

Mr. \& Mrs. Nicholas J. Campbell

Richard L. Campbell

Robert Llewellyn Campbell

Col. \& Mrs. Robert M. Campbell, USA (Ret.)
In Memory of William Elmer \& Bertha Tuttle Campbell

Fauntella Campbell Hill

Clan Campbell Society, Nova Scotia

Patricia \& Michael Coller

Hope \& William Convenience

Glenna C. DuMond

Walter \& Rosemarie Campbell

Dunden

George \& Dorothy Gibbs

James W. \& Patricia R. Hoffstetter

Jean R. Ljungkill

Mr. \& Mrs. Patrick C. MacCulloch

Mr. \& Mrs. Thomas E. Murley

Dr \& Mrs. Thomas J. Quinlan

Ms Dayla Reagan-Buell

Robert C. St. John, Jr

Mr. \& Mrs. James B. Scroggs

Mrs. Clifford Withers Walker 